\title{
The jamming elasticity of emulsions stabilized by ionic surfactants
}

\author{
Frank Scheffold, ${ }^{* c}$ James N. Wilking, $\uparrow^{\mathrm{a}}$ Jakub Haberko, $\star^{\mathrm{c}}$ Frédéric Cardinaux ${ }^{\mathrm{c}}$ \\ and Thomas G. Mason*ab
}

\begin{abstract}
Oil-in-water emulsions composed of colloidal-scale droplets are often stabilized using ionic surfactants that provide a repulsive interaction between neighboring droplet interfaces, thereby inhibiting coalescence. If the droplet volume fraction is raised rapidly by applying an osmotic pressure, the droplets remain disordered, undergo an ergodic-nonergodic transition, and jam. If the applied osmotic pressure approaches the Laplace pressure of the droplets, then the jammed droplets also deform. Because solid friction and entanglements cannot play a role, as they might with solid particulate or microgel dispersions, the shear mechanical response of monodisperse emulsions can provide critical insight into the interplay of entropic, electrostatic, and interfacial forces. Here, we introduce a model that can be used to predict the plateau storage modulus and yield stress of a uniform charge-stabilized emulsion accurately if the droplet radius, interfacial tension, surface potential, Debye screening length, and droplet volume fraction are known.
\end{abstract}

Detailed measurements of the shear rheology of disordered, charge-stabilized, monodisperse, oil-in-water emulsions ${ }^{1}$ have substantially improved the understanding of elasticity, yielding, and flow of soft matter systems. Although concentrated emulsions have been made and enjoyed over many centuries in forms such as mayonnaise and lotion, emulsions fabricated using typical mixers and blenders are typically polydisperse. The variability in polydispersity prevented reliable quantitative predictions of the elastic shear modulus of concentrated

${ }^{a}$ Department of Chemistry and Biochemistry, University of California, Los Angeles, California 90095, USA. E-mail: mason@chem.ucla.edu; Fax: +1 310206 4038; Tel: +13102060828

${ }^{b}$ Department of Physics and Astronomy, University of California, Los Angeles, California 90095, USA

${ }^{c}$ Department of Physics, University of Fribourg, Chemin du Musée 3, 1700 Fribourg, Switzerland. E-mail: Frank.Scheffold@unifr.ch; Fax: +41 26300 9747; Tel: +41 26 3009060

$\dagger$ Present address: Chemical and Biological Engineering Department, Montana State University 306 Cobleigh Hall, Bozeman, MT 59717-3920, USA.

\$ Present address: Faculty of Physics and Applied Computer Science, AGH University of Science and Technology, al. Mickiewicza 30, 30-059 Krakow, Poland. emulsions. ${ }^{2}$ Depletion-induced droplet size segregation, introduced by Bibette $^{3}$ enabled the fractionation of significant quantities of uniform monodisperse emulsions. Subsequently, Mason et al. showed that the elastic shear modulus $G_{\mathrm{p}}$ of nonattractive, microsized emulsion droplets could be well represented over a wide range of droplet volume fractions by the semi-empirical formula: $G_{\mathrm{p}} \simeq 1.6 \phi_{\mathrm{eff}}\left(\phi_{\mathrm{eff}}-\phi_{\mathrm{J}}\right)$. When scaled by the Laplace pressure, $G_{\mathrm{p}}$, a linear property, and the shear stress associated with yielding, $\sigma_{\mathrm{y}}$, a non-linear rheological property, each collapse onto separate master curves if the bare droplet volume fraction $\phi$ is converted into an effective volume fraction $\phi_{\text {eff }}=\phi(1+H / 2 R)^{3}$, where a $\phi$-dependent effective thickness $H \sim$ 5-20 $\mathrm{nm}$ of the interfacial layer, is used to account for screened electrostatic interactions between droplet interfaces. ${ }^{4,5}$ Moreover, in this work the important connection between the measured $\phi_{\mathrm{J}}$ associated with the rapid onset of elasticity towards the Laplace pressure scale and the volume fraction associated with random close packing (RCP) of disordered spheres, $\phi_{\mathrm{J}} \simeq 0.64$ was established. Overall corroboration of the measured $G_{\mathrm{p}}\left(\phi_{\text {eff }}\right)$ came in the form of computer simulations by Lacasse and Grest, which, to within the uncertainties in the experiments and simulations, showed essentially the same dependence as the measurements over a limited range of $\phi_{\text {eff }}$ near and above $\phi_{\mathrm{J}}{ }^{5}$ Importantly, Lacasse's simulations also showed the first example of non-affine motion (i.e. soft spots) of disordered jammed droplets even when the system has been subjected only to very small shear strains. ${ }^{5}$ This leads to a gradual increase in the modulus for $\phi_{\text {eff }}$ above $\phi_{\mathrm{J}}$, rather than a step-jump that was predicted for perfect crystals of droplets. ${ }^{6}$ Later work by O'Hern et al. and others confirmed this important finding using the terminology of jamming, and expanded upon it. ${ }^{7-11}$ Recently we have shown that this jamming-scenario can be applied to quantitatively model the linear and nonlinear elastic properties of micron sized emulsion droplets. ${ }^{12}$

For emulsions of nanoscale droplets, known as nanoemulsions, however, the radius $R$ begins to approach the Debye screening length, $\lambda_{\mathrm{D}}$. In this limit, the electrostatic repulsion, which leads to very large differences between $\phi_{\text {eff }}$ and $\phi$, is not 
properly taken into account by the ad-hoc $H(\phi)$ intended for near-microscale and larger droplets. Such charge-stabilized nanoemulsions, after having been repeatedly fractionated using ultracentrifugation to provide uniform droplet sizes, can become elastic solids at very low droplet volume fractions, even as low as about $\phi \simeq 0.2 .^{1,13}$ To explain the observation of solidification at $\phi$ far below the jamming of hard spheres, Wilking and Mason introduced a simple model of ionically stabilized, disordered, elastic nanoemulsions that successfully connected the average repulsive interaction potential as a function of distance between the droplets to $G_{\mathrm{p}}(\phi) .{ }^{13}$ While this model enabled an accurate measurement of the Debye-screened potential at nanoscopic lengths from macroscopic rheology, their published work did not provide explicit expressions for $G_{\mathrm{p}}$ as a function of $R, \phi, \lambda_{\mathrm{D}}$, oil-water interfacial tension $\gamma$, and electrostatic surface potential $\psi_{0}$ on the droplets.

Given the limitations of the currently available models of emulsion rheology, it would be desirable to combine the interaction potential associated with droplet jamming and deformation developed by Lacasse $e t$ al. with a full treatment of the screened charge repulsion between charged droplets. Such a model could be used to predict the rheology of a wide range of charge-stabilized emulsions having droplet sizes ranging from the macroscale to the nanoscale. Here, we demonstrate that such a quantitative comparison is feasible when taking into account the coupling of the electrostatic potential to the energy associated with the deformation of the oil droplets.

We derive our model for the elasticity of a dense, disordered, monodisperse emulsion from scaling relations suggested in the framework of the jamming scenario for soft spheres having sharp interfaces. ${ }^{\mathbf{8}, 14}$ At the jamming volume fraction $\phi_{\mathrm{J}}$ of soft frictionless spheres a marginally connected solid emerges. Above the transition, the elastic shear modulus depends on the specific pair potential of droplet interaction $E(r)$, where $r$ is the center-to-center separation between droplets, as well as on the connectivity of the stress-bearing network, characterized by the excess number of bonds $\Delta Z=Z-Z_{\mathrm{c}} \sim \sqrt{\varepsilon}$ with $\varepsilon=\phi / \phi_{\mathrm{J}}-1$. The modulus $G_{\mathrm{p}} \sim k \times \Delta Z$ is found to be proportional to the average bond strength or spring constant $k=\partial^{2} E(r) / \partial r^{2}$ and can then be written as: $:^{7-9,12}$

$$
G_{\mathrm{p}}(\phi) \simeq a_{1} \sqrt{\phi_{\mathrm{J}}} \frac{k(\phi)}{\pi R} \times \sqrt{\varepsilon}
$$

Similarly, for the yield stress one can write:

$$
\sigma_{\mathrm{y}}(\phi) \simeq a_{2} \phi_{\mathrm{J}}^{1.2} \frac{k(\phi)}{\pi R} \times \varepsilon^{1.2}
$$

using a density scaling suggested by computer simulations. ${ }^{15,16}$ Estimates for the constants are $a_{1} \simeq 0.25, a_{2} \simeq 0.05$, as reported recently. ${ }^{12,17}$ The jamming contribution due to the excess number of bonds can be isolated when considering the yield strain

$$
\gamma_{\mathrm{y}}=\sigma_{\mathrm{y}} / G_{\mathrm{p}}=\left(a_{2} / a_{1}\right) \phi_{\mathrm{J}}^{0.7} \times \varepsilon^{0.7} \simeq 0.15 \times \varepsilon^{0.7}
$$

For the comparison to experiments we consider silicone oilin-water emulsions stabilized by $10 \mathrm{mM}$ SDS for droplet sizes ranging from $2 R \sim 50-3000 \mathrm{~nm}$. The surfactant concentration is kept high enough to prevent droplet coalescence but also sufficiently low so that micellar depletion attractions are negligible. Oil droplets have been prepared by shear rupturing of a crude emulsion and subsequent size fractionation using. depletion-induced creaming. ${ }^{3}$ The emulsions obtained are uniform $\delta R \sim 10-12 \%$ and we use the particle size as determined by light scattering or microscopy. Nanoscale droplet sizes smaller than $2 R \sim 200 \mathrm{~nm}$ have been obtained by using a high-pressure microfluidic flow device, and, after ultracentrifugal size-fractionation, the resulting emulsions typically show a slightly higher polydispersity of roughly $\delta R / R \sim 20 \% .^{18}$ Some residual polydispersity is advantageous since droplet structures remain disordered. The experimental average for the mean hydrodynamic radius is obtained from dynamic light scattering (DLS). Technical details about sample preparation and the standard procedures employed for measuring the shear modulus and yield stress have been published previously. ${ }^{\mathbf{1 , 4 1 3}}$ However, we want to point out that rheological measurements of the nanoemulsions are more challenging due to the small volumes available. As a consequence we could only extract data for samples with a sufficiently high elastic modulus and moreover the accuracy of the measurements is slightly reduced. The latter mainly affects the yield stress data as the line intersection method on the log-log plot of stress versus strain, which defines the yield point, becomes less precise. ${ }^{4}$ Finally, in our plots we present all available data but note that our model, based on eqn (1) and (2) is valid only in the athermal limit. This means our model description should be compared to data $G_{\mathrm{p}}$ $\gg k_{\mathrm{B}} T / R^{3}$ and $\sigma_{\mathrm{y}} \gg k_{\mathrm{B}} T /(2 R)^{3}$. Entropic contributions will dominate at lower densities and for lower values of $G_{\mathrm{p}}$ and $\sigma_{\mathrm{y} \cdot}{ }^{12,16}$

Due to the presence of electrostatic repulsions jamming is not conditioned by direct physical contact of the droplets. We thus choose the critical interparticle distance for jamming $r_{\mathrm{c}}$ to be equal to the distance when the double layer repulsive interaction potential, $u(h)$ exceeds $c \times k_{\mathrm{B}} T$, where $c$ is a constant factor of order unity and $h$ is the gap distance between the droplet interfaces. From this point on, athermal energetic effects will dominate. We note that this transition can be understood in analogy to the fluid-crystal transition of monodisperse charged spheres having a hard-core. In the latter case, the crystal boundary has been established at $u\left(h_{\mathrm{c}}\right) \simeq k_{\mathrm{B}} T,{ }^{19}$ as long as $\kappa R \geq 10$ and $u(h=0) \gg k_{\mathrm{B}} T$, conditions that are fulfilled for the droplets studied here. For our emulsions crystallization however is suppressed due to droplet polydispersity and therefore the solid phases remain disordered. To this end, we consider the electrostatic interaction energy $u(h)$ of two charge stabilized spherical emulsion droplets, each having diameter $2 R$. We define the center-to-interface distance by $x / 2$. In addition to the Debye layer, we take into account an incompressible interfacial layer, or black film, ${ }^{20,21}$ of thickness $d_{\mathrm{bf}} \simeq 5 \mathrm{~nm}$. The center-to-center distance $r$ of two interacting droplets is thus $r=$ $h+d_{\mathrm{bf}}+x$. Note that the assumptions we make are exactly the same as in the earlier work of Mason et al., ${ }^{4,5}$ the only difference 
being that here we attempt to model explicitly the coupling of electrostatic repulsive and elastic interfacial interactions arising from droplet deformation. For the concentration range addressed here, $\phi \leq 0.85$, shape deformations are small and droplets retain a nearly spherical shape. Therefore, since $R \gg$ $\lambda_{\mathrm{D}}$ and $h \ll 2 R$, the electrostatic double-layer interaction potential $u(h)$ can be written as: $\mathbf{1 9 , 2 2}^{19}$

$$
u(h)=C_{\mathrm{e}} e^{-\kappa h},
$$

where $\kappa^{-1}=\lambda_{\mathrm{D}}=\sqrt{\varepsilon_{\mathrm{r}} \varepsilon_{0} k_{\mathrm{B}} T /\left(2 e^{2} I\right)}$ is the Deybe length and $C_{\mathrm{e}}=2 \pi R \varepsilon_{\mathrm{r}} \varepsilon_{0} \psi_{0}^{2}$ is the contact potential energy. Here $\varepsilon_{0}$ is the vacuum permittivity and $\varepsilon_{\mathrm{r}}=80$ is the static dielectric constant of water. The Debye length $\lambda_{\mathrm{D}}=3.4 \mathrm{~nm}$ is calculated for an electrolyte concentration of $I=8.2 \mathrm{mM}$, equal to the critical micelle concentration of SDS at room temperature. ${ }^{23}$ The only adjustable parameters in our model are $\psi_{0}$ and $c$. We assume that $\psi_{0}$ is independent of $R$ and $I$. Jamming occurs at a volume fraction $\phi_{\mathrm{c}}$ when the value of the interaction potential exceeds the thermal energy at $u\left(h_{\mathrm{c}}\right) \simeq c \times k_{\mathrm{B}} T$ or $h_{\mathrm{c}} \simeq \ln \left(C_{\mathrm{e}} /\left(c k_{\mathrm{B}} T\right)\right) / \kappa$ and thus we can write

$$
\phi_{\mathrm{c}}=\phi_{\mathrm{J}}\left(2 R+d_{\mathrm{bf}}\right)^{3} /\left(2 R+d_{\mathrm{bf}}+h_{\mathrm{c}}(R)\right)^{3}
$$

Similar excluded-volume based arguments have been used in the past to describe the generalized crystallization phase diagram of monodisperse soft spheres. ${ }^{24}$

Next we need to estimate the density dependence of the excess number of bonds $\Delta Z(\phi)$ for the charge stabilized emulsion droplets. As discussed above the droplets jam at $\phi_{\mathrm{c}} \leq \phi_{\mathrm{J}}$ which suggest an initial scaling with $\varepsilon \sim \phi / \phi_{\mathrm{c}}-1$. At higher densities however the Debye layer is compressed and for $\phi \rightarrow 1$ the original scaling $\varepsilon \rightarrow \phi / \phi_{\mathrm{J}}-1$ will be recovered. To account for this we interpolate between the two limiting cases with $\varepsilon_{\text {eff }}=$ $\phi / \phi_{\mathrm{c}}^{\text {eff }}-1$ and $\phi_{\mathrm{c}}^{\text {eff }}=\phi_{\mathrm{c}}+\left(\phi_{\mathrm{J}}-\phi_{\mathrm{c}}\right)\left(\phi-\phi_{\mathrm{c}}\right) /\left(1-\phi_{\mathrm{c}}\right)$. We can check the consistency of this approach by comparison to the experimental values for the shear strain. As shown in Fig. 1 the yield strain data for all droplet sizes, spanning more than an order of magnitude in size, approximately collapse on a master curve that is fairly well described by eqn (3). Here we must stress again the experimental difficulties to measure precise values of the yield stress $\sigma_{\mathrm{y}}$. As can be seen in Fig. 1 this leads to substantial noise in the data for the yield strain $\gamma_{\mathrm{y}} \simeq \sigma_{\mathrm{y}} / G_{\mathrm{p}}$ and thus precludes a more quantitative comparison with the model predictions.

To determine the bond strength $k(\phi)$ we consider the full droplet-droplet interaction potential $E(r)$. Neglecting entropic contributions, this potential can be represented as a sum of the elastic energy associated with deformation of droplet interfaces $V(x)$ and an energy $u(h)$ arising from the compression of the Debye layer: $E(r)=V(x)+u(h)$. The interfacial deformation contribution can be written as ${ }^{5}$

$$
V(x)=0.72 \gamma R^{2}\left[(2 R / x)^{3}-1\right]^{\alpha}
$$

with $\alpha \simeq 2.32 .^{4,5,11}$ The interfacial tension is $\gamma=9.8 \mathrm{mN} \mathrm{m}^{-1}$ (ref. 1 and 4) for poly-dimethylsiloxane silicone oil-water interfaces at a $10 \mathrm{mM}$ sodium dodecyl sulfate (SDS) concentration,

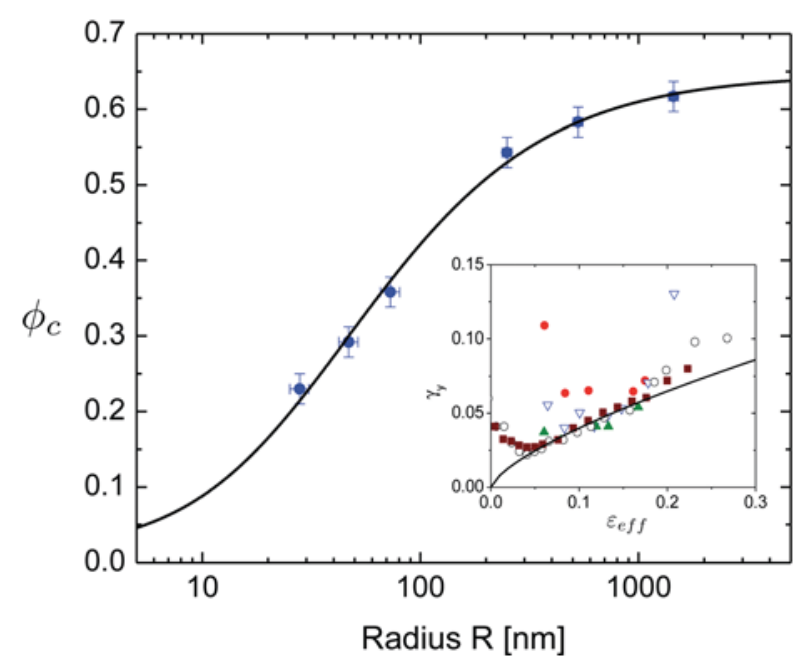

Fig. 1 Jamming volume fraction $\phi_{\mathrm{c}}$ of charge stabilized emulsions as a function of droplet size [ø]. Solid line: best fit of the theory for charged droplets, eqn (5) with $\psi_{0} / \sqrt{ } c=308 \mathrm{mV}$ and $\phi_{J}=0.646$ (for details see text). Inset: estimate for the yield strain $\gamma_{\mathrm{y}} \simeq \sigma_{\mathrm{y}} / G_{\mathrm{p}}$ for different droplet radii $R[\mathrm{~nm}]=28[\mathbf{0}], 47[\nabla], 73[\mathbf{\Delta}], 250[0], 530[\square]$ plotted as a function of the effective excess density parameter $\varepsilon_{\text {eff. }}$. The solid line shows the predictions from eqn (3): $\gamma_{y} \simeq 0.15 \times \varepsilon_{\text {eff }}^{0.7}$.

and the Laplace pressure scale of an undeformed droplet is given by $\gamma / R$.

In order to find the equilibrium condition for a given $r$, we minimize the total energy with respect to $x$ (see also ref. 25). We numerically solve (for fixed $r$ ): $\partial E / \partial x=0$. This calculation provides values for $x$ and also for $h=r-\left(x+d_{\mathrm{bf}}\right)$. As two droplets begin to approach, the soft tail of the double-layer potential is compressed while the core deforms little $(x \simeq 2 R)$. In this regime the bond strength $k(\phi)=C_{\mathrm{e}} \kappa^{2} e^{-\kappa h(\phi)}$ scales exponentially with $h(\phi)$. For higher densities the oil droplet itself will also be deformed and both contributions are coupled and we cannot derive an analytical solution for $k(\phi)$ any more. We note that the average interdroplet distance $r$ is set by the droplet number density which in turn is related to the volume fraction occupied by the oil droplets and thus $\phi \propto r^{-3}$. When the droplets are marginally in contact $r=2 R+d_{\mathrm{bf}}$, which in turn $\operatorname{sets}^{11} \phi(r) \simeq \phi_{\mathrm{J}}\left(\left(2 R+d_{\mathrm{bf}}\right) / r\right)^{3}$.

For the comparison of the model to the experimental data we proceed as follows: we first determine approximate values for $\psi_{0}$ and $c$ and then determine $\phi_{\mathrm{c}}(R)$ by numerically extrapolating the experimental values for the shear modulus $G_{\mathrm{p}}$ to zero using the full expression eqn (1). This procedure is largely insensitive to the exact choice of the guess parameters as long as $c$ is taken of order unity. The values we obtain for $\phi_{\mathrm{c}}$ are shown in Fig. 1. We then fit the $\phi_{\mathrm{c}}$-data using the expression given in eqn (5) and obtain $\psi_{0} / \sqrt{c}=308 \pm 50 \mathrm{mV}$ and $\phi_{\mathrm{J}} \simeq 0.646 \pm 0.01$. The latter value for $\phi_{\mathrm{J}}$ is in good agreement with numerical studies of close packing ${ }^{26}$ that predict $\phi_{\mathrm{J}} \simeq 0.65$ for polydispersities of the order of $10-20 \%$. The fitted value for the surface potential $\psi_{0}$ (modulus a factor $\sqrt{c}$ of order one) is higher compared to the value reported by Calderon et al. ${ }^{22}$ for SDS stabilized octane ferrofluid emulsion droplets in the dilute limit $\left(\psi_{0} \sim 50 \mathrm{mV}\right)$. 
Using a constant value $c \simeq 3 \pm 1$ we obtain excellent agreement between eqn (1) and (2) and a comprehensive set of experimental data for near-microscale and nanoscale ionic emulsions, as shown in Fig. 2 and 3. Although some of the data shown have been obtained from ref. 4 and 13, here, to extend the range of comparison with our model, we also present new data for the yield stress of nanoemulsions as a function of droplet radius and $\phi$, the dependence of $G_{\mathrm{p}}$ on added [ $\left.\mathrm{NaCl}\right]$ and $\phi$ of nanoemulsions, and also $G_{\mathrm{p}}(\phi)$ of uniform microscale droplets larger than previously reported. The new data sets were obtained using exactly the same experimental procedures as the published ones. Fig. 2 displays a linear plot of the concentration dependent shear modulus which allows us to focus on the compressed regime where droplet deformation is important, whereas the logarithmic representation in Fig. 3 reveals more clearly the onset of elasticity near the jamming transition. For nanoemulsions $(2 R<150 \mathrm{~nm})$, rather than using the experimental average for the mean hydrodynamic radius obtained from dynamic light scattering (DLS), we adjust the droplet size for a best fit, albeit staying within the limits of the size uncertainty $\pm 10 \%$. Originally reported DLS values of the radii are also given in parentheses. Moreover, in the inset of (Fig. 3a) we show a data set for the $R=46(47) \mathrm{nm}$ droplet size where an additional 10,40 and $90 \mathrm{mM}$ of electrolyte $\mathrm{NaCl}$ has been added ${ }^{1,27,28}$ resulting in a reduced Debye length of $\lambda_{\mathrm{D}} \simeq 2.3,1.4$ and $1 \mathrm{~nm}$. At electrolyte concentrations of $40 \mathrm{mM}$ and higher eqn (1) qualitatively captures the trend but fails to describe the

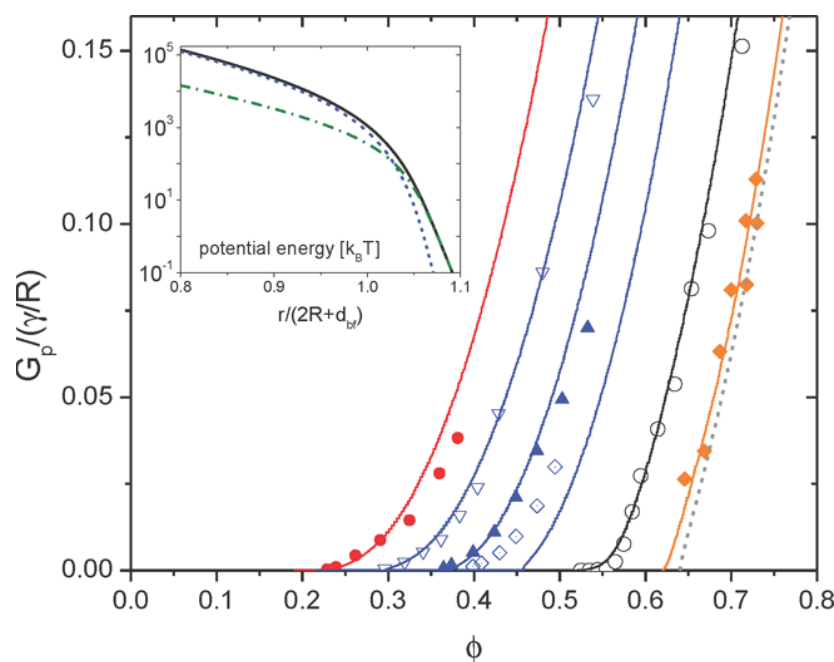

Fig. 2 Elastic shear modulus normalized by the Laplace pressure scale, $G_{p} /(\gamma / R)$, of microscale and nanoscale emulsions for different droplet radii $R[\mathrm{~nm}]=31(28)[\bullet], 46(47)[\nabla], 46(47) 10 \mathrm{mM} \mathrm{NaCl}[\mathbf{\Delta}]$, 46(47) $40 \mathrm{mM} \mathrm{NaCl}[0], 250$ [0], 1450 [0]. The size of the smaller droplets $(2 R<150 \mathrm{~nm}$ ) has been adjusted slightly for a best fit (sizes as denoted in ref. 13 and 18 are given in parentheses). Two data sets were taken for a nanoscale emulsion with $\mathrm{NaCl}$ added to reduce $\lambda_{\mathrm{D}}$. Solid lines: plots derived from eqn (1) with $\psi_{0} / \sqrt{c}=308 \mathrm{mV}, c=3$ and $\phi_{\mathrm{J}}=$ 0.646 . Dotted line: $R / \lambda_{\mathrm{D}} \rightarrow \infty$. Inset: potential energy contributions for droplets having size (diameter) $2 R=500 \mathrm{~nm}$. Solid line: total potentia energy $E(r)$. Dashed line: electrostatic contribution. Dotted line: contribution due to interfacial deformation of the oil droplet. The dimensionless center-to-center distance is denoted by $r /\left(2 R+d_{\mathrm{bf}}\right)$.

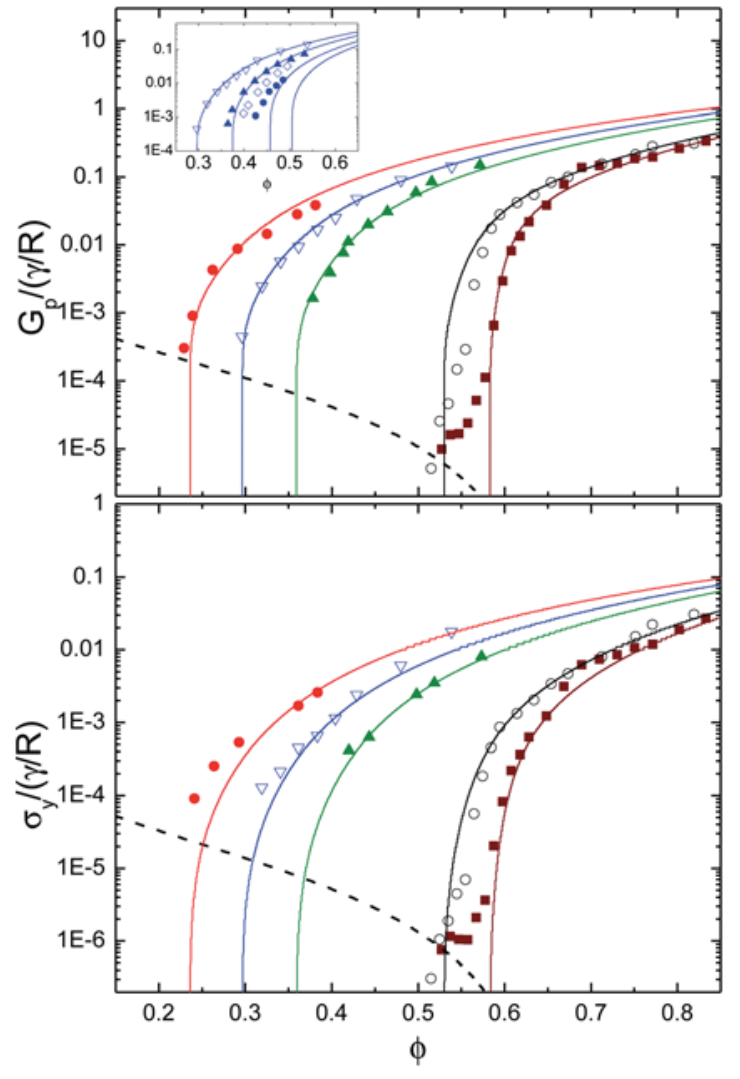

Fig. 3 Shear modulus (a) and yield stress (b) of emulsions above the onset of elasticity. Symbols: experimental data for nano- and microscale emulsions with droplet radius $R[\mathrm{~nm}=31(28)$ [e], 46 (47) [V], 67 (73) [এ], 250 [0], $530[\square]$. Lines: predictions by eqn (1) and (2) with $\psi_{0} / \sqrt{c}=308 \mathrm{mV}, c=3$ and $\phi_{\mathrm{J}}=0.646$. The dashed lines indicate the onset of entropic contributions towards the elastic properties of the emulsions $G_{\mathrm{p}} \sim k_{\mathrm{B}} T / R^{3}$ and $\sigma_{\mathrm{y}} \sim k_{\mathrm{B}} T /(2 R)^{3}$, with $R(\phi)$ taken from Fig. 1. The inset shows the data and theory for the $R=46(47) \mathrm{nm}$ sample for different amounts of added $\mathrm{NaCl}$ (from left to right: $c_{\mathrm{NaCl}}=0,10,40$, $90 \mathrm{mM})$.

experimental data quantitatively. In this limit, eqn (4), is however expected to fail, in line with previous studies of

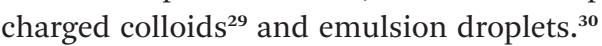

In conclusion, our results demonstrate excellent agreement between experiments and the model. Our results highlight the importance of explicitly taking into account nanoscopic interfacial properties when modeling micron, submicron, and nanoscale colloids. Although the interfacial properties can be system-dependent for different types of colloids, the assumption of effectively hard interactions between colloids is an oversimplification, not only here for ionic emulsions, but we infer also for the vast majority of experimental systems. ${ }^{31}$ Moreover, we could show that the jamming scenario, originally developed primarily for larger particles approaching the granular limit, is also highly relevant for submicron sized particles, where, in principle, entropic contributions are expected to become increasingly important. The latter are generally believed to be driven by the cageing of particles by their peers. ${ }^{32}$ Caging however is dependent on accessible free volume which is again controlled by particle-particle interactions. Thus, we 
anticipate that the model that we have presented could be further improved by including such entropic contributions to the shear modulus of disordered glassy emulsions for droplet volume fractions near and below the jamming point. ${ }^{12,16,32} \mathrm{An}$ important, and very fascinating aspect, will be that in the absence of a sharp interface both the entropically driven glass transition scenario, ${ }^{32}$ due to cageing, and jamming, due to direct interactions, are set by the thermal energy $k_{\mathrm{B}} T$. We speculate that this interplay will lead to a continuous transition across the glassy and jammed regime reminiscent of the unified scenario discussed by Ikeda, Berthier and Sollich ${ }^{16}$ for the case of soft and highly deformable spheres with a sharp interface.

This work was supported by the Swiss National Science Foundation under grant no. 132736 and 149867. JH acknowledges funding from a Sciex Swiss Research Fellowship no. 10.030. T.G.M. and J.N.W. thank UCLA for support.

\section{References}

1 M. M. Fryd and T. G. Mason, Annu. Rev. Phys. Chem., 2012, 63, 493518.

2 H. M. Princen and A. D. Kiss, J. Colloid Interface Sci., 1986, $112,427$.

3 J. Bibette, J. Colloid Interface Sci., 1991, 147, 474-478.

4 T. G. Mason, et al., Monodisperse Emulsions: Properties and uses, in Encyclopedia of Emulsion Technology, ed. P. Becher, Marcel Dekker, New York, Basel, Hong Kong, 1996, p. 299; T. G. Mason, J. Bibette and D. A. Weitz, Phys. Rev. Lett., 1995, 75, 2051; T. G. Mason, J. Bibette and D. A. Weitz, J. Colloid Interface Sci., 1996, 179, 439.

5 M.-D. Lacasse, G. S. Grest, D. Levine, T. G. Mason and D. A. Weitz, Phys. Rev. Lett., 1996, 76, 3448; T. G. Mason, M.-D. Lacasse, G. S. Grest, D. Levine, J. Bibette and D. A. Weitz, Phys. Rev. E, 1997, 56, 3150-3166.

6 D. C. Morse and T. A. Witten, Europhys. Lett., 1993, 22, 549.

7 C. S. O'Hern, L. E. Silbert, A. J. Liu and S. R. Nagel, Phys. Rev. E, 2003, 68, 011306.

8 A. J. Liu, S. R. Nagel, W. van Saarloos, M. Wyart in ref. 14.

9 I. Zaccone and E. Scossa-Romano, Phys. Rev. B, 2011, 83, 184205.

10 B. P. Tighe, Phys. Rev. Lett., 2011, 107, 158303.

11 J. R. Seth, M. Cloitre and R. T. Bonnecaze, J. Rheol., 2006, 50, 353; L. Mohan and R. T. Bonnecaze, Soft Matter, 2012, 8, 4216.

12 F. Scheffold, F. Cardinaux and T. G. Mason, J. Phys.: Condens. Matter, 2013, 25, 502101.

13 J. N. Wilking and T. G. Mason, Phys. Rev. E, 2007, 75, 041407.
14 Dynamical Heterogenity in glasses, colloids and granular matter, ed. L. Berthier, G. Biroli, J.-P. Bouchaud, L. Cipelletti and W. van Saarloos, Oxford University Press, 2011.

15 P. Olsson and S. Teitel, Phys. Rev. Lett., 2007, 99, 178001; P. Olsson and S. Teitel, Phys. Rev. E: Stat., 2011, 83, 030302, we note that slightly different scaling exponents, between 1 and 1.5, for harmonic spheres, have also been reported in the literature, for a discussion we refer to ref. 12 .

16 A. Ikeda, L. Berthier and P. Sollich, Phys. Rev. Lett., 2012, 109, 018301; A. Ikeda, L. Berthier and P. Sollich, Soft Matter, 2013, 9, 7669-7683.

17 For simplicity, we have neglected a weak concentration dependence $\propto \phi$ that follows from general statistical mechanical considerations. ${ }^{9}$ Moreover, we have not taken into account an additional weak concentration dependence of $r$ and $\alpha$ in the jammed state., ${ }^{5,11}$ The latter has been shown to increase from $\alpha=2.23 \rightarrow 2.47$ over the range of interest. ${ }^{5}$.

18 T. G. Mason, J. N. Wilking, K. Meleson, C. B. Chang and S. M. Graves, J. Phys.: Condens. Matter, 2006, 18, R635.

19 A.-P. Hynninen and M. Dijkstra, Phys. Rev. E: Stat., 2003, 68, 021407.

20 D. Exerowa, D. Kashiev and D. Platikanov, Adv. Colloid Interface Sci., 1992, 40, 201.

21 O. Belorgey and J. J. Benattar, Phys. Rev. Lett., 1991, 66, 313.

22 F. Leal Calderon, T. Stora, O. Mondain Monval, P. Poulin and J. Bibette, Phys.Rev. Lett., 1994, 72, 2959-2962.

23 J. Marra and M. L. Hair, J. Colloid Interface Sci., 1989, 128, 511.

24 A. P. Gast and W. B. Russel, Phys. Today, 1998, 51, 24-30.

25 G. Romeo and M. Pica Ciamarra, Soft Matter, 2013, 9, 54015406.

26 R. S. Farr and R. D. Groot, J. Chem. Phys., 2009, 131, 244104; C. B. O. Donovan, E. I. Corwin and M. E. Möbius, Philos. Mag., 2013, 93(31-33), 4030-4056.

27 The addition of $\mathrm{NaCl}$ has shown to have no effect on the critical micelle concentration of SDS. ${ }^{28}$

28 I. M. Umlong and K. Ismail, Colloids Surf., A, 2007, 299, 8-14. 29 S. H. Behrens, D. I. Christl, R. Emmerzael, P. Schurtenberger and M. Borkovec, Langmuir, 2000, 16, 2566-2575.

30 J. Bibette, T. G. Mason, H. Gang, D. A. Weitz and P. Poulin, Langmuir, 1993, 9, 3352-3356.

31 C. P. Royall, W. C. K. Poon and E. R. Weeks, Soft Matter, 2013, 9, 17-27.

32 L. Cipelletti and E. R. Weeks in ref. 14. 